\title{
Seguridad y eficacia de la utilización de levosimendán sin dosis de carga en una unidad de falla cardiaca especializada
}

\author{
Safety and effectiveness of levosimendan \\ administration without loading dose in a \\ specialized heart failure unit
}

\author{
Juan Manuel Senior, Clara Inés Saldarriaga, \\ Julián Miguel Aristizábal,Jairo Alonso Rendón • Medellín (Colombia)
}

\section{Resumen}

Objetivo: evaluar la eficacia y seguridad del uso de levosimendán en pacientes con falla cardiaca severa crónica descompensada con signos evidentes de bajo gasto, e indicación de utilización de inotrópico, utilizando el medicamento sin dosis de carga, con una mayor concentración y en infusión continua hasta completar la dosis de 12.5 miligramos en total.

Metodología: estudio retrospectivo, descriptivo. Se evaluaron los cambios en la presión arterial media, desarrollo de arritmias o muerte durante la infusión y clase funcional I (NYHA) antes y dos meses después de recibir el medicamento.

Resultados: se observó cambio en la clase funcional durante el seguimiento a dos meses. La infusión del medicamento fue bien tolerada y no se observaron efectos adversos significativos durante la hospitalización. La presión arterial media antes de la infusión fue $71 \pm 21.6$ vs $75 \pm 20.9 \mathrm{mmHg} 24$ horas después, y $71 \pm 21.6$ vs $71.6 \pm 21.4 \mathrm{mmHg} 48$ horas después. Ningún paciente presentó arritmias inducidas por el inotrópico y se presentó solamente una muerte (4.7\%) por sepsis.

Conclusiones: el presente reporte es una experiencia inicial que sugiere que la administración de la infusión de levosimendan sin bolo inicial disminuye la incidencia de hipotensión y arritmias en los pacientes con falla cardiaca y puede emplearse en un servicio de hospitalización diferente a la Unidad de Cuidado Intensivo con seguridad (Acta Med Colomb 2011: 36: 68-72).

Palabras clave: insuficiencia cardiaca, terapéutica, efectividad, agentes cardiotónicos.
Abstract
Objective: to evaluate the safety and effectiveness of levosimendan administration in patients with chronic heart failure, signs of low output, and indications for inotropic therapy. The protocol included a continuous infusion of levosimendan without loading dose, with a total dosage of 12.5 miligrams.
Methods: a retrospective, descriptive study was performed to evaluate changes in mean arterial pressure, the development of arrhythmias, adverse effects like death during the infusion, and functional changes in the two months after the treatment.
Results: the infusion was well tolerated, without side effects. Mean arterial pressure before the infusion was $71 \pm 21.6$ vs. $75 \pm 20.9 \mathrm{mmHg} 24$ hours after and $71 \pm 21.6$ vs. $71.6 \pm 21.448$ hours after. There was one death (4.7\%), explained by non-cardiovascular causes (sepsis). An improvement in functional class was observed during the follow-up.
Conclusion: this report of an initial experience suggests that the administration of levosimendan infusion without loading dose is safe and can be used outside the intensive care unit (Acta Med Colomb 2011: 36: 68-72).
Key words: heart failure, therapeutics, cardiotonic agents, effectiveness.
Dr. Juan Manuel Senior: FACP, Cardiólogo Intervencionista, Clínica de Falla Cardiaca, Universidad de Antioquia, Hospital Univer- sitario San Vicente de Paúl; Dra. Clara inés Saldarriaga: Cardióloga Clínica, Clínica Cardiovascular Santa María, Profesora de la Sección de Cardiología, Universidad de Antioquia; Dr. Julián Miguel Aristizábal: Cardiólogo, Fellow de Electrofisiología Universidad CES; Dr. Jairo Alonso Rendón: Cardiólogo Ecocardiografista, Universidad de Antioquia, Medellín (Colombia). Correspondencia. Dr. Juan Manuel Senior. Hospital Universitario San Vicente de Paúl, Universidad de Antioquia. Medellín, Antioquia.
E-mail: mmbt@une.net.co
Recibido: 03/II/2011 Aceptado: 14/IV/2011 
El síndrome de falla cardiaca se caracteriza por la activación de diversos mecanismos hemodinámicos y neurohumorales, que mejoran en forma inicial la función ventricular posterior a una injuria miocárdica. El enfoque terapéutico inicial, en pacientes con disfunción ventricular izquierda severa al administrar inotrópicos para mejorar la contractilidad demostró efectos benéficos hemodinámicos y en la sintomatología; sin embargo, se relacionó con aumento en la incidencia de arritmias ventriculares, hipotensión y aumento de la mortalidad, especialmente en el grupo de pacientes con cardiopatía dilatada de etiología isquémica $(1,2)$. Este efecto se ha atribuido a múltiples factores, entre ellos el aumento del calcio intracelular, del consumo miocárdico de oxígeno, la taquicardia, la depleción de fosfatos de alta energía, activación neurohormonal y a la generación de arritmias, entre otros. En contraposición a la orientación simplista de la utilización de inotrópicos, el bloqueo neurohormonal ha producido los mejores resultados en cuanto a mejoría en calidad de vida, disminución del proceso de remodelación ventricular y de la mortalidad.

Durante la última década, la investigación se ha orientado hacia medicamentos con un mecanismo de acción que no incluya el aumento de los niveles intracelulares de calcio y que en su lugar contribuyan a mejorar la respuesta contráctil con niveles normales de éste. Este nuevo grupo de medicamentos inotrópicos se ha denominado "sensibilizadores del calcio" y se han descrito tres tipos de acuerdo con el mecanismo de acción, entre ellos los que aumentan la afinidad del calcio por la troponina como el pimobendan, los que prolongan los cambios estructurales del complejo troponina inducidos por el calcio como el levosimendan y los que tienen efecto directo sobre los puentes de actina- miosina (3). El metabolismo del levosimendan es complejo, su eliminación se hace a través de la conjugación hacia derivados de cisteína y cisteinglicina, generando metabolitos activos como el OR-1896, cuya vida media es de 77 a 81 horas (3). Se han descrito otros efectos importantes como la inducción de vasodilatación arterial sistémica y coronaria, a través de la acción sobre canales de potasio sensibles a ATP, logrando incrementar la contractilidad sin aumentar el consumo de oxígeno, mejorar la función diastólica, aumentar el flujo sanguíneo coronario actuando como medicamento antiisquémico, tiene además la capacidad de disminuir la poscarga y los fenómenos de apoptosis $(4,5)$. El levosimendan se une a la porción $\mathrm{N}$ terminal de la troponina $\mathrm{C}$ en una forma calciodependiente, lo que estabiliza el complejo troponina C-calcio, que a su vez reduce la inhibición de la troponina I sobre la actina-miosina ATPasa (5). Los resultados de los estudios iniciales en humanos demostraron un efecto benéfico en pacientes con falla cardiaca moderada a severa; sin embargo, se reportó una mayor incidencia de hipotensión, arritmias, tales como fibrilación auricular, hipocaliemia y cefalea (5). La incidencia de taquicardia ventricular es discretamente mayor al compararlo con placebo (6-8), pero no en la comparación con dobutamina. Los estudios
RUSSLAN, LIDO y CASINO corroboraron estos resultados y mostraron una tendencia a disminuir la mortalidad en el seguimiento, lo que convertía al medicamento en una droga bastante atractiva en el contexto del tratamiento de la falla cardiaca crónica severa descompensada (6-8). Con base en estos resultados se diseñó un estudio multicéntrico, aleatorizado, doble ciego para comparar el efecto del levosimendan con dosis de carga e infusión por 24 horas en la mortalidad a 180 días, comparado con dobutamina. El estudio no demostró diferencias significativas en mortalidad a los 180 días; sin embargo, se encontraron diferencias en desenlaces secundarios tales como disminución de los niveles de péptido natriurético cerebral, entre otros, con un buen perfil de seguridad en cuanto a efectos adversos (9). El análisis juicioso del estudio nos arroja algunas conclusiones interesantes tales como la alta tasa de hipotensión luego de la administración de la dosis de carga, efecto esperado al utilizar una droga con acción vasodilatadora directa en pacientes con falla cardiaca severa con cifras de presión arterial limítrofes por el bajo gasto (9), La disminución de las cifras de presión arterial y la inducción de arritmias podría oscurecer el efecto benéfico del medicamento, máxime teniendo en cuenta las disminuciones de los niveles de péptido natriurético cerebral de la población. Existió además una tendencia a una mejor respuesta clínica en pacientes con historia de falla cardiaca crónica que en pacientes con falla cardiaca aguda, con una baja tasa de eventos adversos y efecto neutro en la mortalidad. Estas consideraciones motivaron la realización de un estudio abierto, de seguimiento, de los pacientes con falla cardiaca severa crónica descompensada de la Clínica de Falla Cardiaca del Hospital Universitario San Vicente de Paúl, con signos evidentes de bajo gasto, con indicación de utilización de inotrópico, sobre la utilización del medicamento sin dosis de carga, con una mayor concentración y en infusión continua hasta completar la dosis de 12.5 miligramos en total.

\section{Método}

Estudio retrospectivo, descriptivo que incluyó los pacientes con falla cardiaca crónica descompensada severa con síntomas de bajo gasto, mayores de 18 años, clasificación de Stevenson B o C (10), sin respuesta a diuréticos o vasodilatadores, que recibieron infusión de levosimendan en la unidad de falla cardiaca del Hospital Universitario San Vicente de Paúl.

El protocolo de administración de modificó de la siguiente manera: el medicamento viene en ampollas de $2.5 \mathrm{mg} /$ $\mathrm{mL}$ por $5 \mathrm{~mL}$, se diluyó en $250 \mathrm{~mL}$ de DAD $5 \%$, se omitió la dosis de carga e inmediatamente se inició en infusión continua a $0.05 \mu \mathrm{g} / \mathrm{kg} / \mathrm{min}$ y se tituló progresivamente en forma rápida de acuerdo con la tolerancia según cifras de presión arterial a $0.1 \mu \mathrm{g} / \mathrm{kg} / \mathrm{min}$ hasta $0.2 \mu \mathrm{g} / \mathrm{kg} / \mathrm{min}$, administrando completamente la ampolla. Se continuó el manejo convencional para falla cardiaca con inhibidores de la enzima convertidora de angiotensina o bloqueadores de los 
receptores de angiotensina, y betabloqueadores, de acuerdo con las guías (11) y la tolerancia. Se excluyeron los pacientes menores de 18 años, con falla cardiaca aguda, los pacientes con indicación de soporte ventilatorio o requerimiento de monitoreo hemodinámico invasivo con catéter en arteria pulmonar, obstrucción del tracto de salida del ventrículo izquierdo, arritmias letales, presión arterial sistólica menor de $80 \mathrm{mmHg}$, utilización previa de otro inotrópico durante la hospitalización actual u otra comorbilidad que indicara su ingreso a la unidad de cuidados intensivos. Los desenlaces evaluados fueron: cambios en la presión arterial media, desarrollo de arritmias o muerte durante la infusión y clase funcional I (NYHA) antes y dos meses después de recibir el medicamento. También se evaluaron las características demográficas de los pacientes, la etiología de la falla cardiaca, fracción de eyección y el uso concomitante de otros inotrópicos.

El análisis estadístico incluyó el cálculo de porcentajes, media y la desviación estándar.

\section{Resultados}

Se incluyeron 21 pacientes, $81.25 \%$ hombres $(n=17)$ y $18.75 \%(n=4)$ mujeres, con edad promedio de $48 \pm 16$ años. La causa más frecuente de la falla cardiaca fue la cardiopatía no isquémica en $81.2 \%(\mathrm{n}=17)$ y $18.7 \%$ tenían cardiopatía isquémica $(n=4)$. Dentro de la etiología no isquémica la enfermedad valvular fue la más frecuente $(33.33 \%)$, seguida de la cardiomiopatía dilatada idiopática $(28.6 \%)$, el diagnóstico de cardiopatía idiopática se realizó luego de excluir otras causas como enfermedad coronaria, valvular y enfermedad de Chagas. La fracción de eyección promedio fue de $30 \% \pm$ 13.26. Las características clínicas se describen en la Tabla 1.

El levosimendan se inició a $0.05 \mu \mathrm{g} / \mathrm{kg} / \mathrm{min}$ y se tituló rápidamente hasta alcanzar $0.2 \mu \mathrm{g} / \mathrm{kg} / \mathrm{min}$ en todos los pacientes. El tiempo promedio de duración de la infusión fue de $26.6 \pm 5.1$ horas. El 19\% de los pacientes recibieron un segundo bolo de levosimendan durante el periodo de seguimiento. Respecto al uso de otros medicamentos con efecto inotrópico, $37.5 \%$ requirieron otro inotrópico entre ellos milrinone $(25 \%)$ y dobutamina $(6.25 \%)$ durante la hospitalización; $25 \%$ requirieron soporte vasopresor con dopamina en $12.5 \%$ o norepinefrina en $12.5 \%$ y $56.25 \%$ recibieron digoxina. Se observó cambio en la clase funcional durante el seguimiento a dos meses. Antes de recibir la infusión $37.5 \%$ de los pacientes se encontraban en clase funcional NYHA III y $62.5 \%$ en clase IV, dos meses después $12.5 \%$ se encontraban en NYHA I, $12.5 \%$ en II, $50 \%$ en III y $25 \%$ en NYHA IV.

La infusión del medicamento fue bien tolerada y no se observaron efectos adversos significativos durante la hospitalización. La presión arterial media antes de la infusión fue $71 \pm 21.6$ vs $75 \pm 20.9 \mathrm{mmHg} 24$ horas después, y $71 \pm 21.6$ vs $71.6 \pm 21.4 \mathrm{mmHg} 48$ horas después (Tabla 2). Ningún paciente presentó arritmias inducidas por el inotrópico y se presentó solamente una muerte $(4.7 \%)$ intrahospitalaria re-
Tabla 1. Características clínicas de los pacientes que recibieron infusión de levosimendan sin bolo en una unidad de falla cardiaca especializada.

\begin{tabular}{|l|r|}
\hline Características clínicas & Porcentaje (\%) \\
\hline Hombres & 81.25 \\
Edad & $48 \pm 16$ \\
Hipertensión & 28.6 \\
Diabetes & 5 \\
Dislipidemia & 10 \\
Arritmia & 14.2 \\
Cardiopatía isquémica & 18.8 \\
Cardiopatía no isquémica & 81.2 \\
Clase functional NYHA III & 37.5 \\
Clase functional NYHA IV & 62.5 \\
Ingurguitación yugular & 95.2 \\
Crépitos pulmonares & 52.4 \\
S3 & 19 \\
Edemas & 66.66 \\
FE\% & \\
\hline FE: Fracción de expulsión & $30 \pm 13.26^{*}$ \\
$*$ Media y desviación estandar. & \\
\hline
\end{tabular}

Tabla 2. Presión arterial antes, durante y después de la infusión de levosimendan.

\begin{tabular}{|l|c|c|c|}
\hline $\begin{array}{c}\text { Presion arterial } \\
(\text { PA) } \mathbf{~ m m H g}\end{array}$ & Antes & $\begin{array}{c}\text { 24 horas } \\
\text { después }\end{array}$ & $\begin{array}{c}\text { 48 horas } \\
\text { después }\end{array}$ \\
\hline PA sistólica & $93.15 \pm 13.81$ & $97.5 \pm 23.27$ & $95 \pm 27.8$ \\
PA diastólica & $62.5 \pm 14.3$ & $65 \pm 10.54$ & $60 \pm 12.6$ \\
PA MEDIA & $71 \pm 21.6$ & $75 \pm 20.9$ & $71.6 \pm 21.4$ \\
\hline
\end{tabular}

lacionada con sepsis. En los dos meses de seguimiento cinco $(23.8 \%)$ pacientes requirieron una nueva hospitalización y seis fallecieron $(28.6 \%)$. De los pacientes que fallecieron, dos habían recibido un segundo bolo de levosimendan por persistencia de los síntomas de bajo gasto y necesidad de continuar en terapia con medicación inotrópica.

\section{Discusión}

El levosimendan es un medicamento inotrópico nuevo para el tratamiento de la falla cardiaca crónica severa descompensada con signos de bajo gasto y hasta la fecha es el único que ha demostrado producir mejoría sintomática sostenida que persiste aún después de su aplicación sin afectar la mortalidad (6). Otros agentes inotrópicos y vasodilatadores han sido relacionados con aumento en la mortalidad. Nuestros resultados concuerdan con lo reportado previamente en la literatura, demostrando una mejoría franca en la clase funcional hasta dos meses después de la aplicación del medicamento según la clasificación de Nueva York (NYHA), resaltando la importancia de impactar no sólo la supervivencia sino la calidad de vida en una patología crónica con gran compromiso funcional, laboral, social y familiar, por lo que consideramos que este es un desenlace relevante. 
La duración sostenida del efecto de levosimendan se explica por la acción de su metabolito altamente activo, el OR 1896, que alcanza su concentración máxima en plasma dos días después de terminarse la infusión y presenta efectos hemodinámicos similares que pueden durar hasta siete a nueve días después de suspender la infusión (12).

Existen varios aspectos importantes para resaltar respecto a la administración del medicamento en este estudio: la no utilización de dosis de carga evitó la generación de inestabilidad hemodinámica o al menos la hipotensión asociada a éste, la dosis de la infusión titulada de levosimendan que utilizamos $(0.05 \mu \mathrm{g} / \mathrm{kg} / \mathrm{min})$ es menor a la reportada en la literatura mundial $(9,12)$ y por esta razón se incrementó el tiempo de duración de la infusión, lo que explicaría la menor frecuencia de efectos adversos, entre ellos la hipotensión que fue reportada en $16 \%$ de los pacientes en el estudio SURVIVE vs $14 \%$ en el grupo de dobutamina (5). Este efecto adverso limita su uso en los pacientes en choque cardiogénico o con bajo gasto cardiaco con cifras de presión arterial limítrofes y parece estar relacionado con la administración de la dosis de carga, explicando por qué en el presente estudio no se presentaron modificaciones significativas en la presión arterial media. La presencia de arritmias es otro de los efectos secundarios que ha cuestionado la seguridad del medicamento, es así como la presencia de fibrilación auricular fue reportada en $6 \%$ de los pacientes que recibieron levosimendan en el estudio SURVIVE y a su vez $8 \%$ presentaron taquicardia ventricular (9), este efecto adverso fue mayor que en el grupo de dobutamina pero menor que en el grupo de placebo. Respecto a los efectos electrofisiológicos de levosimendan, Singh et al (13) reportaron a partir de sus observaciones en pacientes sanos y con falla cardiaca que existe una prolongación modesta del intervalo QT corregido, además de un acortamiento del periodo refractario efectivo de la aurícula y el ventrículo, con dosis altas del medicamento. Esto explicaría parcialmente por qué no encontramos la presencia de arritmias como un efecto adverso significativo de la administración del medicamento a dosis bajas y sin bolo. La dilución en $250 \mathrm{~mL}$ y no en $500 \mathrm{~mL}$, como está recomendado por el laboratorio farmacéutico productor del medicamento, disminuye la posibilidad de empeorar la sobrecarga de volumen, especialmente en pacientes con falla cardiaca crónica descompensada en estadio B o C de Stevenson (10). A pesar de su evidente efecto benéfico en una población seleccionada de una clínica de falla cardiaca, se debe resaltar que esta patología tiene un alto impacto sobre la calidad de vida y la supervivencia, encontrando una frecuencia de reingresos hospitalarios de $23.8 \%$ y mortalidad de $28.6 \%$ a dos meses de seguimiento en el presente reporte.

Teniendo en cuenta que las indicaciones de tratamiento en unidad de cuidados intensivos del síndrome de falla cardiaca crónica severa descompensada son cada vez menores, es relevante resaltar que el tratamiento de estos pacientes en una unidad de falla cardiaca especializada con monitoreo continuo no invasivo es seguro y eficaz. El uso del medicamento a las dosis evaluadas y en un lugar de estas características facilita su aplicación y permite que esté disponible para más pacientes sin la limitación de la disponibilidad de una cama en la unidad de cuidados intensivos. Es importante recalcar que la facilidad de su administración y su perfil de seguridad no justifica el uso indiscriminado del medicamento y éste debe ser reservado para pacientes con claras indicaciones de soporte inotrópico, con las características descritas en nuestro estudio y en la literatura científica.

Las limitaciones del estudio son múltiples teniendo en cuenta que desde el punto de vista metodológico no se realizó asignación aleatoria, el tamaño de la muestra fue pequeño, no se tuvo grupo control, el seguimiento fue corto y el desenlace no se evaluó en forma enmascarada (cegamiento); a pesar de esto consideramos que es una experiencia inicial exitosa, que debe ser evaluada en un estudio a mayor escala con mejor diseño metodológico, por el beneficio que representa para los pacientes con falla cardiaca descompensada que requieren un inotrópico. Específicamente en pacientes con falla cardiaca crónica descompensada con previo uso de betabloqueadores, quienes aparentemente son los pacientes que mejor responden a su utilización (14). En otros escenarios clínicos, aunque existen reportes alentadores, no hay evidencia sólida y objetiva que permita su recomendación (9,15-17).

\section{Conclusión}

El levosimendan representa una alternativa para el tratamiento de los pacientes con falla cardiaca crónica descompensada que requieren soporte con agentes inotrópicos, dentro de este grupo de medicamentos es el que cuenta con más respaldo desde el punto de la evidencia clínica por estudios de adecuada calidad metodológica. El presente reporte es una experiencia inicial que sugiere que la administración de la infusión de levosimendan, sin bolo inicial, disminuye la incidencia de hipotensión y arritmias en los pacientes con falla cardiaca y puede emplearse en un servicio de hospitalización diferente a la unidad de cuidado intensivo con gran seguridad. Esta estrategia debe evaluarse en un estudio multicéntrico con asignación aleatoria con tamaño de muestra adecuado.

\section{Conflicto de intereses}

Los autores del estudio declaran no tener conflictos de interés, el estudio se realizó con recursos propios de los investigadores.

\section{Referencias}

1. Cuffe MS, Califf RM, Adams KF Jr, Benza R, Bourge R, Colucci WS et ál. Outcomes of a Prospective Trial of Intravenous Milrinone for Exacerbations of Chronic Heart Failure (OPTIME-CHF) Investigators. JAMA 2002; 287: 1541-7.

2. Abraham WT, Adams KF, Fonarow GC, Costanzo MR, Berkowitz RL, LeJemtel TH, et ál. In-hospital mortality in patients with acute decompensated heart failure requiring intravenous vasoactive medications: an analysis from the Acute Decompensated Heart Failure National Registry (ADHERE). J Am Coll Cardiol 2005 46: 57-64.

3. Lehtonen L. Levosimendan: a calcium sensitizing agent for the treatment of patients with decompensated heart failure. Current Heart Failure Reports 2004, 1: $136-44$. 
4. Papp Z, Csapó K, Pollesello P, Haikala H,E’des I. Pharmacological mechanisms contributing to the clinical efficacy of levosimendan. Cardiovascular Drug Rev 2005, 23: 71-98

5. Figgit DP, Gillies PS, Goa KL. Levosimendan. Drugs 2001; 61: 613-27.

6. Moiseyev VS, Poder P, Andrejevs N, Ruda MY, Golikov AP, Lazebnik LB et ál. Safety and efficacy of a novel calcium sensitizer, levosimendan, in patients with left ventricular failure due to an acute myocardial infarction. A randomized, placebo-controlled, double blind study RUSSLAN. Eur Heart J 2002; 18: 1422-32.

7. Follath F, Cleland JG, Just H,Papp JG, Scholz H, Peuhkurinen K, et ál. Efficacy and safety of intravenous levosimendan compared with dobutamine in severe low output heart failure LIDO: a randomized double blind trial. Lancet 2002; 360: 196-202.

8. Zairis MN,Apostolatos C,Anastasiadis P, et ál. The effect of calcium sensitizer oral or an inotrope or none in chronic low output decompensated heart failure: results from the calcium sensitizer or inotrope or none in low output heart failure study CASINO. Abstract presented at the annual scientific sessions of the American College of Cardiology. New Orleans March 7-10, 2004.

9. Mebazaa A, Nieminen MS, Packer M, Cohen-Solal A, Kleber FX, Pocock SJ, et al. Levosimendan vs dobutamine for patients with acute decompensated heart failure: the SURVIVE Randomized Trial. JAMA 2007; 297: 1883-91.

10. Nohria A, Tsang SW, Fang JC, Lewis EF, Jarcho JA, Mudge GH, et ál. Clinical assessment identifies hemodynamic profiles that predict outcomes in patients admitted with heart failure. J Am Coll Cardiol 2003 21; 41: 1797-804
11. Heart Failure Society of America, Lindenfeld J, Albert NM, Boehmer JP, Collins SP, Ezekowitz JA, Givertz MM, et ál. HFSA 2010 Comprehensive Heart Failure Practice Guideline. J Card Fail 2010; 16: 1-194.

12. Singh BN, Lilleberg J, Sandell EP, Ylonen V, Lehtonen L, Toivonen L. Effects of levosimendan on cardiac arrhythmia: electrophysiologic and ambulatory electrocardiographic findings in phase II and phase III clinical studies in cardiac failure. Am J Cardiol 1999; 83: 16-20.

13. Mebaza A, Nieminen M, Filippatos G, Cleland JG, Salon JE, Thakkar R et ál. Levosimendan vs Dobutamine outcomes for acute heart failure patients on B-blockers in SURVIVE. Eur J Heart Failure 2009; 11: 304-11.

14. Petaja L, Sipponen J, Hammainen P, Eriksson HI, Salmenperä MT, Suojaranta-Ylinen RT. et ál. Levosimendan reversing low output syndrome after heart transplantation. Ann Thorac Surg 2006; 82: 1529-31.

15. Fuhrmann JT, Schmeisser A, Schulze M, Wunderlich C, Schoen SP, Rauwolf T et ál. Levosimendan is superior to enoximone in refractory cardiogénico shock complicating acute myocardial infarction. Crit Care Med 2008; 36: 2257-66.

16. De Hert S, Lorsomradee S, Cromheecke S, Van der Linden P. The effects of levosimendan in cardiac surgery patients with poor left ventricular function. Anesth Analg 2007; 104: 766-73.

17. Bollen Pinto B, Rheberg S, Ertmer C, Westphal M. Role of levosimendan in sepsis and septic shock. Curr Opin Anesthesiol 2008; 21: 168-77. 